\title{
Article
}

\section{Complementary Fourier Single-Pixel Imaging}

\author{
Dong Zhou ${ }^{1}$, Jie Cao ${ }^{1, *}$, Huan Cui ${ }^{1}$, Qun Hao ${ }^{1}$, Bing-kun Chen ${ }^{1}$ and Kai Lin ${ }^{2}$ \\ 1 Key Laboratory of Biomimetic Robots and Systems, School of Optics and Photonics, Beijing Institute of \\ Technology, Beijing 100081, China; 3120195343@bit.edu.cn (D.Z.); 3120190619@bit.edu.cn (H.C.); \\ qhao@bit.edu.cn (Q.H.); chenbk@bit.edu.cn (B.-k.C.) \\ 2 Beijing Fisheries Research Institute, Beijing 100068,CChina; linkai_bit@126.com \\ * Correspondence: caojie@bit.edu.cn
}

check for

updates

Citation: Zhou, D.; Cao, J.; Cui, H.; Hao, Q.; Chen, B.-k.; Lin, K

Complementary Fourier Single-Pixel Imaging. Sensors 2021, 21, 6544.

https://doi.org/10.3390/s21196544

Academic Editor: Ming-Jie Sun

Received: 1 September 2021

Accepted: 28 September 2021

Published: 30 September 2021

Publisher's Note: MDPI stays neutral with regard to jurisdictional claims in published maps and institutional affiliations.

Copyright: (c) 2021 by the authors. Licensee MDPI, Basel, Switzerland. This article is an open access article distributed under the terms and conditions of the Creative Commons Attribution (CC BY) license (https:/ / creativecommons.org/licenses/by/ $4.0 /)$.

\begin{abstract}
Single-pixel imaging, with the advantages of a wide spectrum, beyond-visual-field imaging, and robustness to light scattering, has attracted increasing attention in recent years. Fourier singlepixel imaging (FSI) can reconstruct sharp images under sub-Nyquist sampling. However, the conventional FSI has difficulty balancing imaging quality and efficiency. To overcome this issue, we proposed a novel approach called complementary Fourier single-pixel imaging (CFSI) to reduce the number of measurements while retaining its robustness. The complementary nature of Fourier patterns based on a four-step phase-shift algorithm is combined with the complementary nature of a digital micromirror device. CFSI only requires two phase-shifted patterns to obtain one Fourier spectral value. Four light intensity values are obtained by loading the two patterns, and the spectral value is calculated through differential measurement, which has good robustness to noise. The proposed method is verified by simulations and experiments compared with FSI based on two-, three-, and four-step phase shift algorithms. CFSI performed better than the other methods under the condition that the best imaging quality of CFSI is not reached. The reported technique provides an alternative approach to realize real-time and high-quality imaging.
\end{abstract}

Keywords: single-pixel imaging; Fourier patterns; complementary nature; phase-shift algorithm

\section{Introduction}

Conventional optical imaging systems generally use a pixelated array of detectors as the light detection unit. Since the theory of ghost imaging (GI) was demonstrated by using quantum-entangled photon pairs in 1995 [1], a bucket detector or a single-pixel detector has gradually been applied in optical imaging systems [2-10]. Single-pixel imaging (SPI), originating from GI, provides scene information by correlating the modulated light patterns generated by a pseudo-thermal source, digital micromirror device (DMD), or other types of spatial light modulators with the intensity of light from the target scene. SPI has the advantages of a wide spectrum, beyond-visual-field imaging and robustness to light scattering, and has been adopted in many fields, including 3D imaging [11-14], terahertz imaging [15-17], multispectral imaging [18-20] and scattering medium imaging [21-23]. However, SPI requires a large number of illumination patterns and takes much time [24]. Therefore, achieving a balance between imaging quality and efficiency remains a challenge in the use of SPI.

The current methods for improving the SPI efficiency are mostly concerned with designing different modulate light patterns and applying compressive sensing theory [25-27]. The different types of modulate light patterns in SPI are random pattern, Hadamard pattern [28], and Fourier pattern [25]. Hadamard and Fourier patterns belong to deterministic orthogonal basis patterns, which are different from non-orthogonal random patterns. Compared to conventional SPI random patterns, Hadamard singlepixel imaging (HSI) and Fourier single-pixel imaging (FSI) have the advantages of using under-sampled data to reconstruct a sharp image and realize perfect reconstruction in 
principle. Further, FSI has better imaging efficiency than HSI [27]. In 2015, Zhang et al. [25] presented FSI based on a four-step phase-shift algorithm that can illuminate a scene with phase-shifting sinusoidal-structured light patterns and applies the inverse fast Fourier transform (IFT) algorithm to reconstruct the final image according to the Fourier spectrum. FSI based on a four-step phase-shift algorithm performs the required full sampling measurements, which is two-times the number of pixels of the illumination pattern. In 2017, Zhang et al. [27] proposed an FSI based on three-step phase-shift algorithm that only requires 1.5-times the number of pixels of the illumination pattern. In 2019, Deng et al. [29] proposed an FSI based on a two-step phase-shift algorithm and demonstrated that it can perform full sampling measurements equivalent to the number of the pixels of the illumination pattern. However, the three- and two-step phase-shift algorithms sacrifice noise robustness to reduce the number of measurements. The differential measurement of the four-step phase-shift algorithm has good noise robustness, but it requires a large number of measurements.

To balance the imaging efficiency and imaging quality, we proposed a novel method based on the complementary nature of DMD and a four-step phase-shift algorithm, that is complementary Fourier single-pixel imaging (CFSI). DMD can be individually oriented at $\pm 12^{\circ}$ with respect to the plane of the array and corresponds to " 0 " and " 1 " in the binary pattern. The area of the pattern where " 1 " locates is naturally complementary to the area where " 0 " locates. The complementary nature of DMD was proposed by Luo et al. [30], and the signal-to-noise ratio of GI using complementary random patterns and Gerchberg-Saxton-like algorithm increases, compared with using conventional random patterns. Ye et al. [31] presented the complementary nature in CGI-based ghost-differenceimaging (GDI) in 2021. They demonstrated multiwavelength-difference GDI and positiondifference GDI by Hadamard-based complementary patterns. Multiwavelength-difference GDI can identify more interested object information and position-difference GDI can directly extract edges in a single-round detection. In FSI, based on the four-step phase-shift algorithm, complementarity exists between patterns of phase-shift 0 and $\pi, \pi / 2$, and $3 \pi / 2$. In contrast to conventional FSI, CSFI has high efficiency of FSI based on two-step phaseshift algorithm and noise robustness of FSI based on four-step phase-shift algorithm, which can considerably improve FSI performance. Compared to conventional FSI, CFSI is able to achieve better imaging quality because it can obtain more Fourier spectral value with the same number of measurements, while having the advantage of robustness to noise. This technology provides an approach to realize real-time and high-quality imaging.

In this paper, we present the principles of CFSI and demonstrate its advantages using simulations and experiments. We compare the proposed method with the current FSI based on two, three, and four-step phase-shift algorithms and finally summarize the whole work and draw conclusions.

\section{Principles of CFSI}

The principles of CFSI are based on conventional FSI using a four-step phase-shift algorithm. CFSI illuminates the object with phase-shifting sinusoidal-structured light patterns, and the reflected or transmitted light from the object is collected by a singlepixel detector to obtain the Fourier spectrum. The final image of the object is obtained by applying the fast IFT algorithm to the Fourier spectrum.

In FSI based on a four-step phase-shift algorithm, four sinusoid patterns with a phase-shift of $\pi / 2$ are used to modulate light. $P^{1}{ }_{\theta}$ represents the pattern of phase-shift $\theta$ with the micromirrors of state " 1 ". Figure 1a shows that the two patterns whose phases differ by $\pi$ are complementary, which is similar to the complementary nature of DMD. As shown in Figure 1b, a beam of surface light illuminates the plane of the DMD vertically, and micromirrors of state " 0 " and " 1 " reflect the light at a deviation of $\pm 24^{\circ}$ from the plane normal. Therefore, modulated light with a phase-shift of 0 in the vertical reflection direction of micromirrors of state " 1 " is obtained, while in the vertical reflection direction of micromirrors of state " 0 ", modulated light with a phase-shift of $\pi$ is obtained. This 
complementary regular also applies to Fourier patterns with a phase-shift of $\pi / 2$ and $\pi 3 / 2$. The illumination pattern $P^{d}{ }_{\theta}$ is expressed as follows:

$$
P_{\theta}^{d}\left(x, y ; f_{x}, f_{y}\right)=a+b \cos \left(2 \pi f_{x} x+2 \pi f_{y} y+\theta\right) .
$$

where $a$ is a DC term and represents the average intensity of the image; $b$ represents the contrast; $f_{\mathrm{x}}$ and $f_{\mathrm{y}}$ represent the spatial frequency; $x$ and $y$ represent the $2 \mathrm{D}$ Cartesian coordinates in the scene; $\theta$ represents the phase parameter; and $d$ is 0 or 1 depending on the state of " 0 " or " 1 " of DMD. The patterns are shown in Figure 1c, and the patterns of $P^{1}{ }_{\theta}$ or $P^{0}{ }_{\theta}$ should be generated and illuminated. Therefore, the values of $\theta$ are equal to 0 (or $\pi$ ) and $\pi / 2$ (or $3 / 2 \pi$ ), respectively. The number of patterns for full sampling required by CFSI is half of the four-step phase-shift FSI, two thirds of the three-step phase-shift FSI, and equal to the two-step phase-shift FSI. The obtained intensity values after the projection of patterns on the object are expressed as:

$$
I_{\theta}^{d}\left(f_{x}, f_{y}\right)=\iint_{\Omega} O(x, y) P_{\theta}^{d}\left(x, y ; f_{x}, f_{y}\right) d x d y
$$

where $\Omega$ is the illumination area and $O(x, y)$ is the object to be imaged. $I^{d}{ }_{\theta}$ represents the light intensity obtained by the projection of $P^{d}{ }_{\theta}$. The Fourier spectrum of $O(x, y)$ can be obtained by processing $I_{0}^{1}, I_{0}^{0}, I^{1} \pi / 2$, and $I_{\pi / 2}^{0}$. The formula is expressed as

$$
2 b \cdot F\{O(x, y)\}=\left[I^{1}{ }_{0}\left(f_{x}, f_{y}\right)-I^{0}{ }_{0}\left(f_{x}, f_{y}\right)\right]+j\left[I^{1}{ }_{\pi / 2}\left(f_{x}, f_{y}\right)-I^{0}{ }_{\pi / 2}\left(f_{x}, f_{y}\right)\right] .
$$

where $F\{O(x, y)\}$ is the Fourier spectrum, and $j$ is the imaginary unit. Although twophase-shifted patterns are generated for illumination, Equation (3) also realizes differential measurement in a symmetric manner for removing $a$. Compared with FSI based on a twoor three-step phase-shift, the proposed method can effectively eliminate the noise caused by environmental illumination. Finally, the final image $O^{\prime}(x, y)$ is obtained by IFT. The formula is expressed as

$$
2 b \cdot O^{\prime}(x, y)=F^{-1}\left\{\left[I^{1}{ }_{0}\left(f_{x}, f_{y}\right)-I^{0}{ }_{0}\left(f_{x}, f_{y}\right)\right]+j\left[I^{1}{ }_{\pi / 2}\left(f_{x}, f_{y}\right)-I^{0}{ }_{\pi / 2}\left(f_{x}, f_{y}\right)\right]\right\}
$$

where $F^{-1}$ represents the IFT operator. Natural Fourier basis patterns are grayscale and cannot be directly loaded into the DMD. In previous research, two typical solutions are used to binarize the grayscale Fourier patterns. One is spatial dithering strategy, and the other is temporal dithering strategy [24,32]. As shown in Figure 2b, the patterns of temporal dithering strategy are successive binary patterns that are decomposed from the grayscale Fourier pattern denoted as B0, B1, and B7. These successive binary patterns are illuminated for different times, and patterns of higher order take more time to illuminate. This temporal dithering strategy is used to approximately replace a grayscale pattern with eight binary patterns. Although grayscale FSI is realized by the temporal dithering strategy, it requires a large amount of illumination and takes much time for sampling. Figure $2 \mathrm{c}$ shows that spatial dithering can accelerate the speed of image acquisition of FSI at the expense of the imaging spatial resolution. A grayscale pattern is replaced by a binary pattern processed by the spatial dithering strategy. FSI based on the spatial dithering strategy is binary FSI. In this paper, we used binary and grayscale FSI to verify CFSI performance through simulations and used binary FSI to verify CFSI through experiments. The spatial dithering strategy used here is the Floyd Steinberg dithering strategy. 
(a)

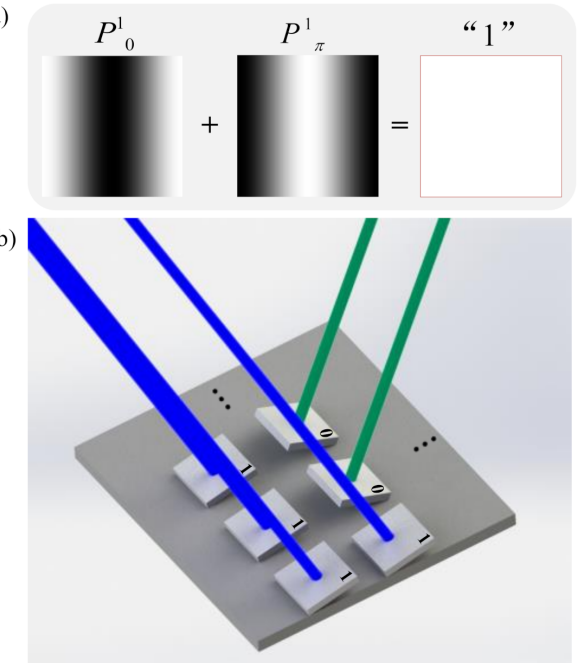

(c)

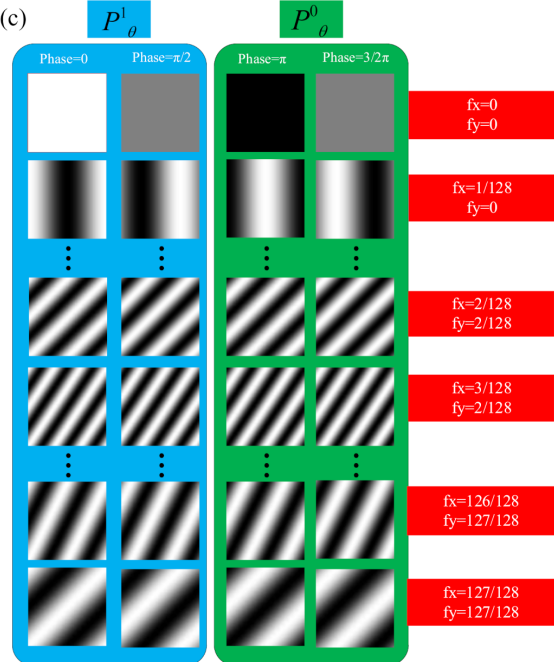

Figure 1. Principles of CFSI. (a) Two patterns whose phase differs by $\pi$ are complementary. (b) Schematic diagram of generating complementary illumination. Two modulated reflected light whose phase-shifts differ by $\pi$ can be obtained when DMD is loaded with one pre-set pattern. (c) Complementary Fourier patterns. Only $P_{\theta}^{1}$ is loaded to DMD, and the light intensity from the target scene of $P^{0}{ }_{\theta}$ can be obtained based on complementary nature.

(a)

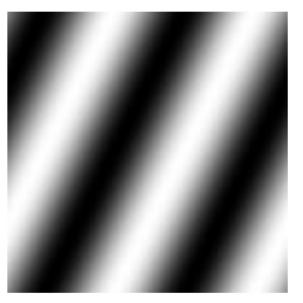

Original grayscale pattern (b)

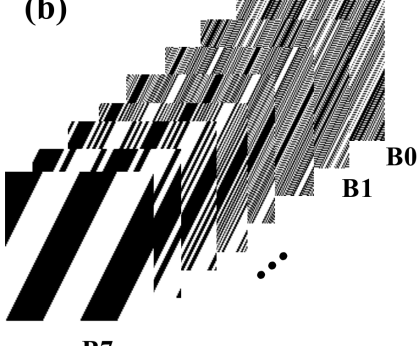

B7

Figure 2. Methods to binarize the grayscale Fourier patterns. (a) original grayscale Fourier pattern, (b) binary Fourier pattern binarized by temporal dithering, and (c) binary Fourier pattern binarized by spatial dithering.

In binary CFSI, we also theoretically analyzed the feasibility of complementary patterns instead of patterns with a phase-shift difference of $\pi$. Figure 3 shows that the complementary patterns are similar to the patterns with a phase-shift difference of $\pi$ but are not exactly the same. The cause of this phenomenon is the quantization error when converting grayscale images to binary images using the spatial dithering strategy. Thus, CFSI is not exactly a two-step transformation of FSI based on a four-step phase-shift algorithm. 
(a)

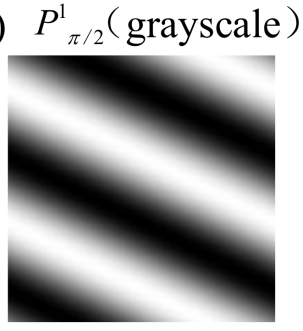

(b)

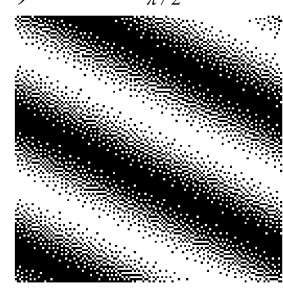

(d)

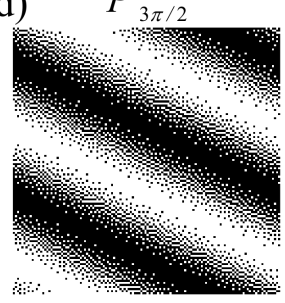

(c) $\quad P_{\pi / 2}^{0}$

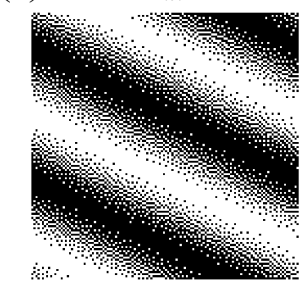

(e)

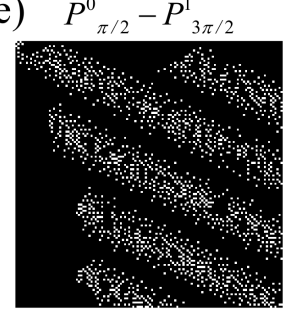

Figure 3. Comparison of complementary binary pattern and phase-shift difference $\pi$ binary pattern. (a) grayscale original Fourier basis pattern with phase-shift $\pi / 2\left(f_{\mathrm{x}}=1 / 128, f_{\mathrm{y}}=126 / 128\right)$. (b) binary Fourier basis pattern binarized from the original $\mathrm{P}_{\pi / 2}^{1}$ with spatial dithering algorithm. (c) complementary pattern of binary $\mathrm{P}_{\pi / 2}^{1}$. (d) phase-shift difference $\pi$ pattern of binary $\mathrm{P}_{\pi / 2}^{1}$. (e) difference of complementary binary pattern and phase-shift difference $\pi$ binary pattern.

\section{Simulations and Experiments}

\subsection{Simulations}

Simulations using grayscale and binary patterns were performed separately to evaluate the performance of CFSI compared with FSI based on two-, three-, and four-step phase-shift algorithms.

The performance of the final reconstructed image was compared quantitatively using the peak signal-to-noise ratio (PSNR) [33] as the evaluation index:

$$
\left\{\begin{array}{l}
\mathrm{PSNR}=10 \log _{10} \frac{\left(2^{k}-1\right)^{2}}{\mathrm{MSE}} \\
M S E=\frac{1}{M} \sum_{x, y}\left(O^{\prime}(x, y)-O(x, y)\right)^{2}
\end{array}\right.
$$

where $M S E$ is the mean square error, $M$ is the number of the pixels of the whole image, and $k$ is the number of bits set as 8. PSNR can reflect the imaging quality; the higher the PSNR, the better the imaging quality.

\subsubsection{Grayscale Patterns}

The reconstruction was simulated using the tested image "cameraman" whose size is $128 \times 128$ pixels. We compared four different methods under the condition that the number of measurements are 600, 1200, 1800, 2400, 3000, 3600, 6400, and 16,384. For CFSI and a two-step phase-shift FSI, full sampling was achieved when the number of measurements reached 16,384. Figures 4 and 5 show that the imaging quality improves with the increase in number of measurements. The PSNR of CFSI was higher than that of the three other methods, and the two-step phase-shift FSI was slightly better than the three-step phaseshift FSI. Conventional FSI based on a four-step phase-shift has the worst performance for requiring four illuminations to achieve one spectrum value. 


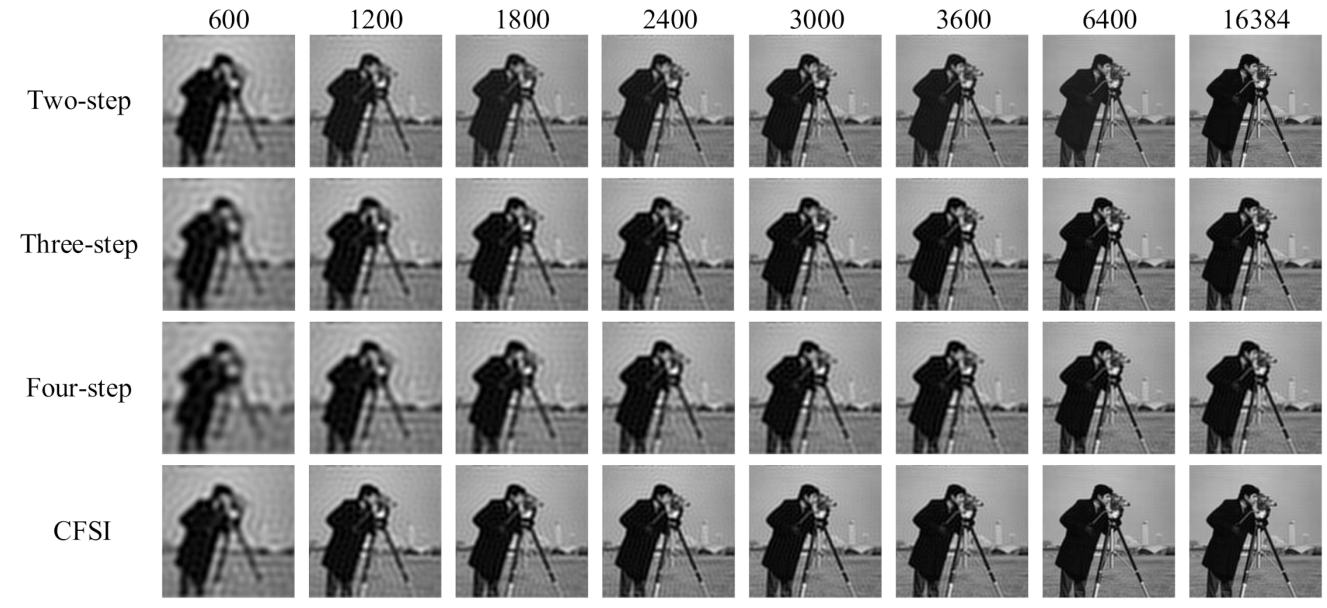

Figure 4. Reconstructed images of grayscale CFSI and two-, three- and four-step phase-shift grayscale FSI under different number of measurements.

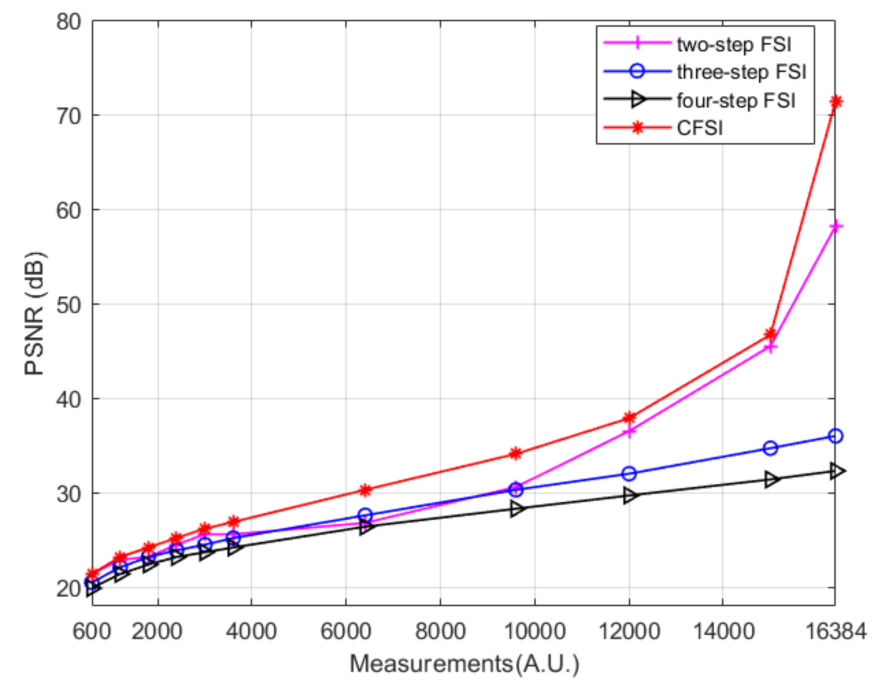

Figure 5. PSNR values of grayscale CFSI and two-, three-, and four-step phase-shift grayscale FSI under different number of measurements.

The above results were obtained under the condition of no noise. However, in the actual application environment, noise, such as ambient light and circuit current, is usually introduced into the imaging system. Therefore, we performed another simulation and added measurement noise to evaluate the performance of the four methods. The measurement noise set was Gaussian white noise shown as:

$$
N(c)=\frac{1}{\sqrt{2 \pi} \sigma} \exp \left(-\frac{c-\mu^{2}}{2 \sigma^{2}}\right)
$$

where $c$ is the noise, $\mu$ is the average value, and $\sigma$ is the standard deviation. We set $\mu$ as 0 and set $\sigma$ as $0,0.1,0.5,1,3,5$, and 10 . Number of measurements was set as 3000. The corresponding reconstructed results are shown in Figure 6, indicating that the imaging quality of CFSI is better than that of the three other methods under the condition of existing noise, except when the noise level is high enough. The FSI based on a two-step phaseshift is most affected by noise because it is not a differential measurement. The PSNR difference of CFSI is higher than that of the four-step phase-shift FSI because CFSI doubles the spectrum value and introduces twice the noise, which is 1.5 times compared with the three-step phase-shift FSI. 
(a)
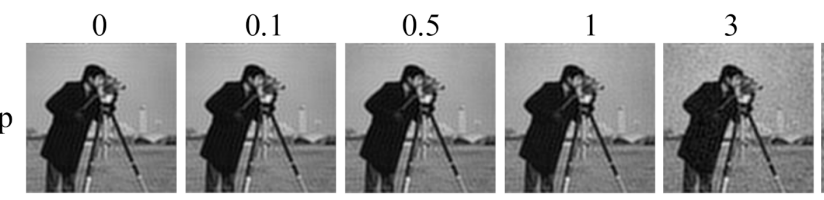
5

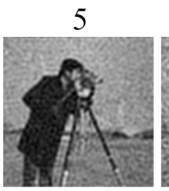

10
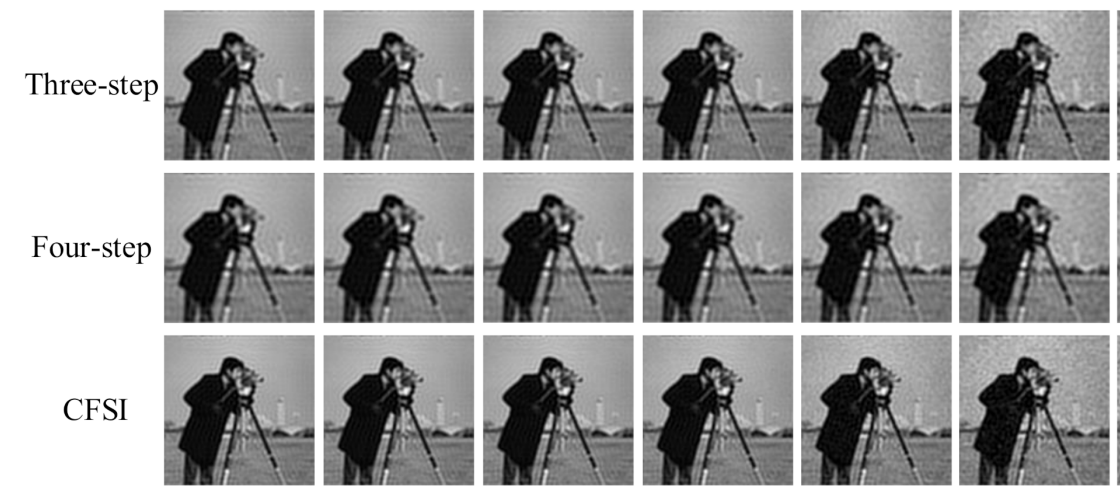

(c)

(b)

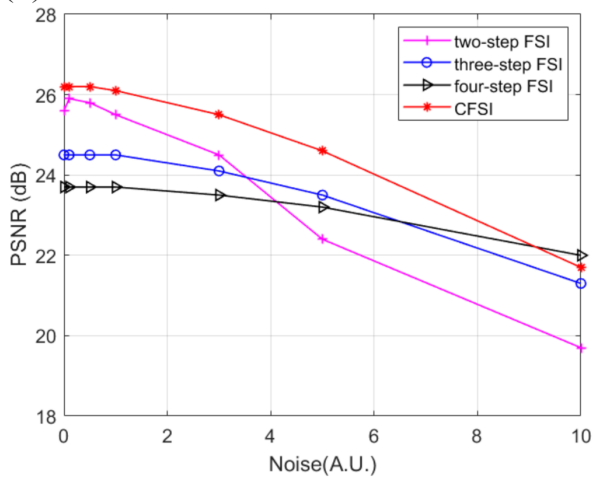

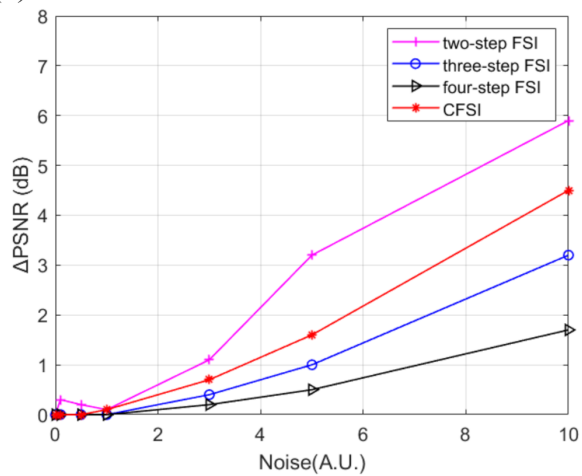

Figure 6. Reconstructed results of grayscale CFSI and two-, three-, and four-step phase-shift FSI under different standard deviation noise. (a) final images of the four methods under different standard deviation noise. (b) PSNR values of the final images. (c) PSNR difference between final images and noiseless images.

\subsubsection{Binary Patterns}

We also performed simulations on FSI with binary patterns based on the spatial dithering strategy. The setup was the same as that of applying grayscale patterns. The reconstructed results without noise are shown in Figures 7 and 8, indicating that the results are different from those obtained by applying grayscale patterns. Given the quantization errors and reduction in the imaging spatial resolution caused by spatial dithering strategy [32], the imaging quality is worse than that of the grayscale FSI. The imaging quality of the two-step phase-shift FSI is worse than that of the three other methods because its base pattern is not applicable after spatial dithering [29]. Qualitative comparison indicates that as the number of measurements increase, the PSNR values of binary CFSI and threeand four-step phase-shift binary FSI increases first and then decreases. CFSI is the first to achieve the best imaging quality, and before that, the PSNR of CFSI is better than the threeand four-step phase-shift binary FSI. 


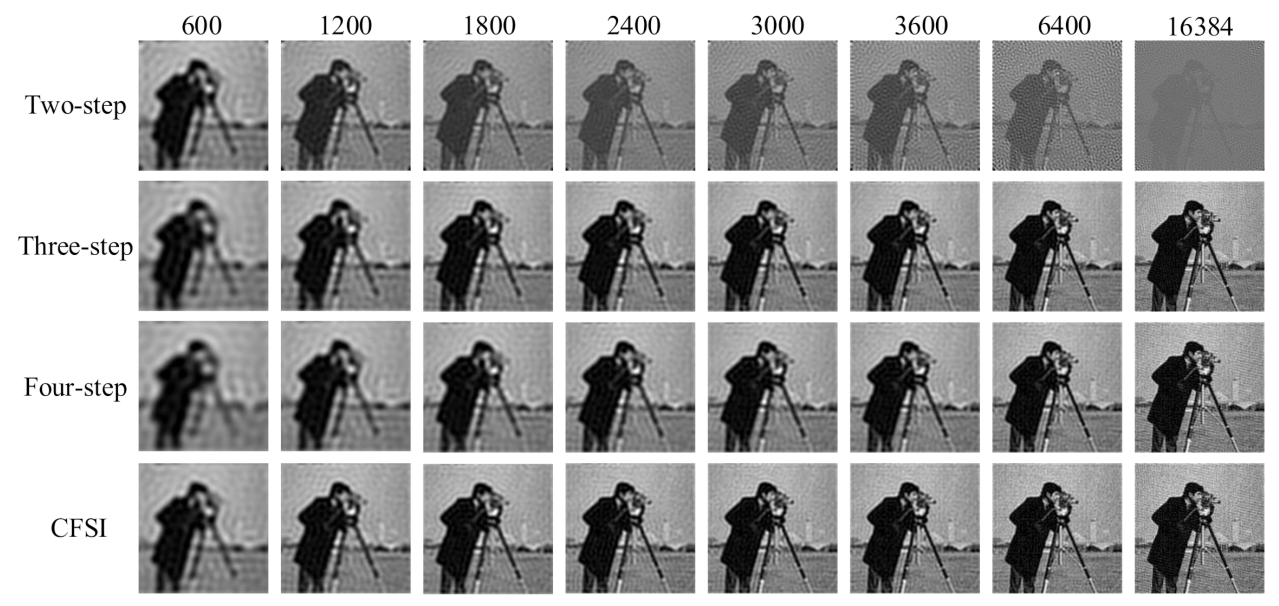

Figure 7. Reconstructed images of binary CFSI and two-, three-, and four-step phase-shift binary FSI under different number of measurements.

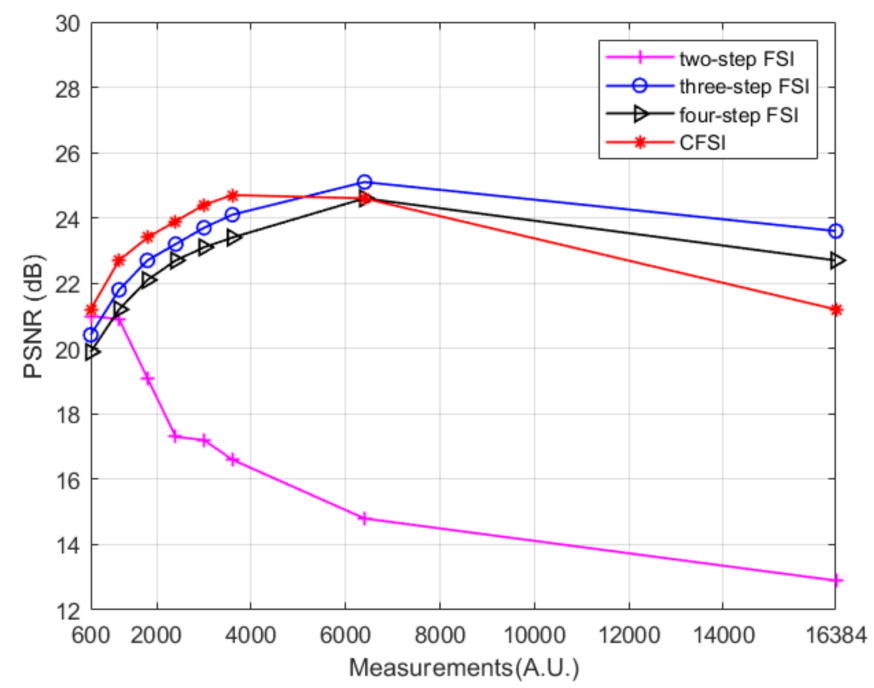

Figure 8. PSNR values of binary CFSI and two-, three-, and four-step phase-shift binary FSI under different number of measurements.

The reconstructed results with noise are shown in Figure 9. Given the poor imaging quality of the two-step phase-shift FSI, the interference caused by noise is lower than that when grayscale patterns were applied; thus, the PSNR difference becomes the smallest. Although the PSNR difference of CFSI is larger than that of the three- and four-step phaseshift FSI, the PSNR value of CFSI is higher than the two other methods under the condition that standard deviation of noise is below 5 . 

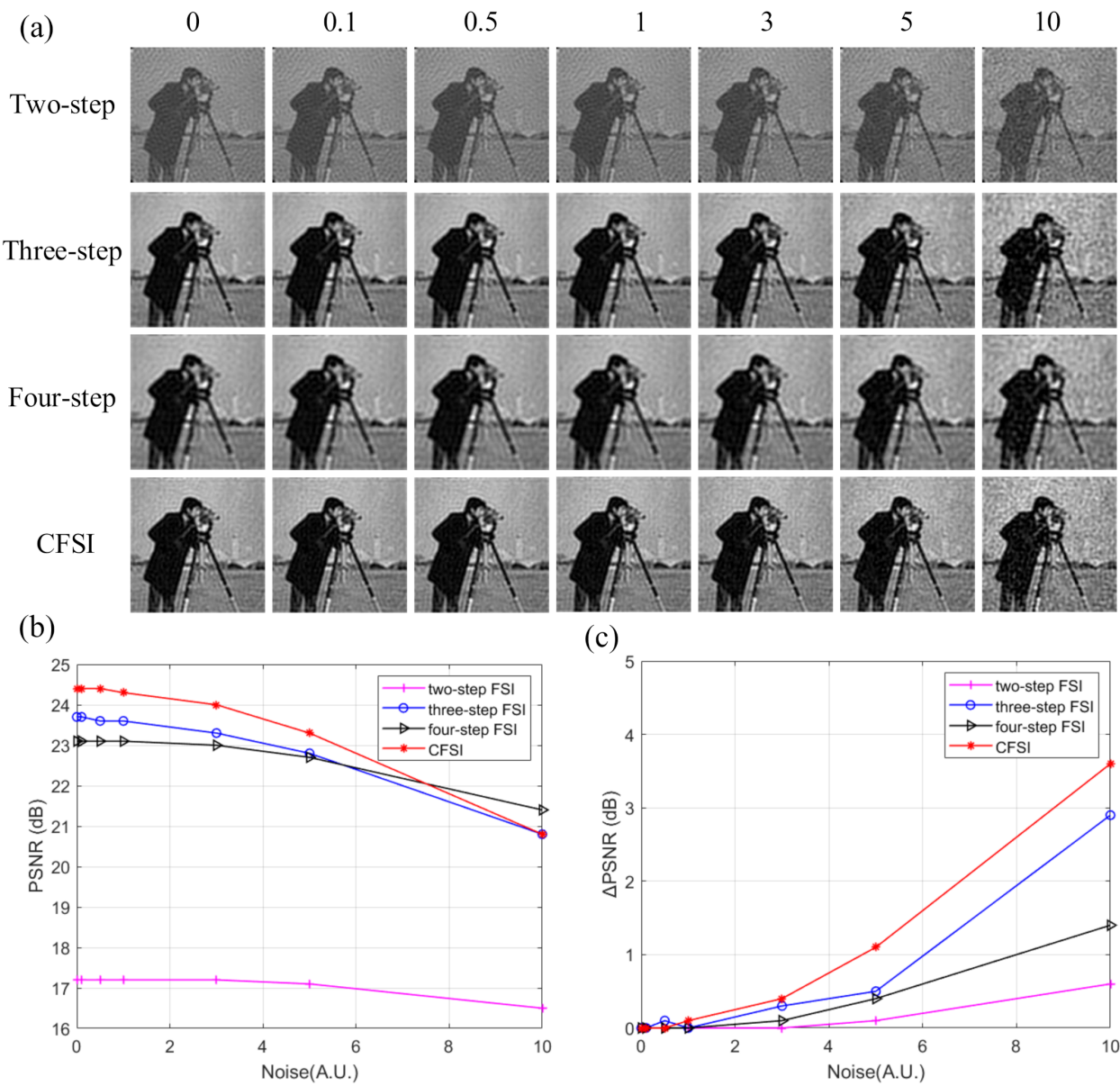

(c)

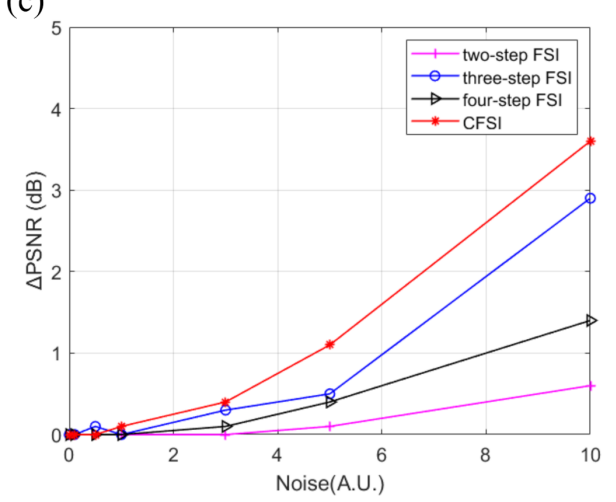

Figure 9. Reconstructed results of binary CFSI and two-, three-, and four-step phase-shift binary FSI under different standard deviation noise. (a) Reconstructed images of the four methods under different standard deviation noise, (b) PSNR values of the final images, and (c) PSNR difference between the final images and noiseless images.

\subsection{Experiments}

The experimental setup is shown in Figure 10. The setup consists of three parts, namely, the illumination system, the detection system, and the object. The illumination system consists of a light-emitting diode operating at 400-760 nm (@20W), DMD (Texas Instruments DLP Discovery 4100 development kit), and lens. The DMD of the DLP development kit consists of $1024 \times 768$ micromirrors, and its maximum binary modulation rate is up to $22 \mathrm{KHz}$. The focal length of the lens is $150 \mathrm{~mm}$. The detection system consists of two photodetectors (Thorlabs PDA36A, active area of $13 \mathrm{~mm}^{2}$ ) and collecting lens of focal length $5 \mathrm{~mm}$, data acquisition board (Gage CSEG8, sampling at $1 \mathrm{MS} / \mathrm{s}$ ), and a computer.

Grayscale FSI with temporal dithering strategy will considerably prolong the sampling time and consume much storage space of DMD. Besides, environmental noise is inevitable in the experimental environment. Although we carried out the experiments in a confined space, when the light illuminated on the target surface and the other blank area, the reflected light fills the entire experimental space due to the large divergence angle of white light. Thus, we performed experiments by adding ambient light noise from light source without adding extra noise. The level of ambient light noise depends on the working power of the light source. During our whole experiments, the light source works at the maximum power. Before the experimental results are shown, some settings should be illustrated first. The resolution of DMD is $1024 \times 768$, which is not suitable for applying $128 \times 128$ pixels binary patterns if all micromirrors are used. We combined $6 \times 6$ micromirrors as one cell, and the patterns occupied part of DMD with $768 \times 768$ resolution. In FSI based on two-, three-, and four-step phase-shift algorithms, patterns with the remaining part of DMD 
set as 0 can be used for sampling. However, in CFSI, the $768 \times 768$-pixel binary Fourier patterns were placed at the middle of the DMD, and the remaining two $768 \times 128$ parts on both sides were occupied by random patterns. As such, the interference of the unused part of the DMD can be eliminated through differential CFSI measurement.

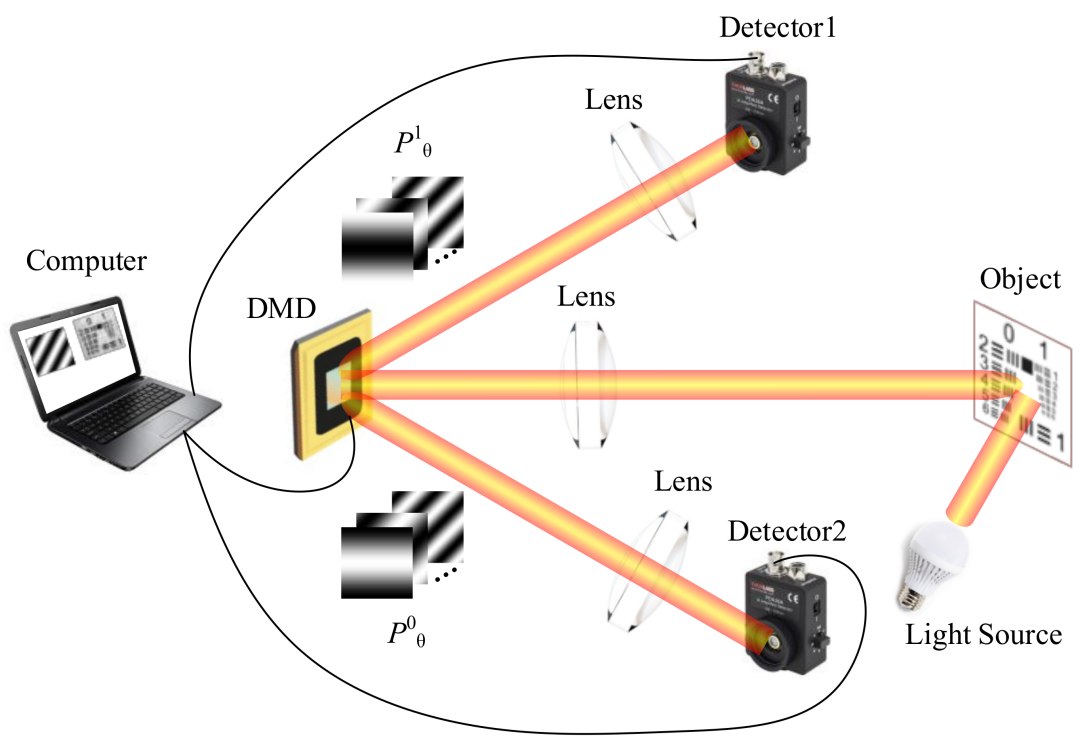

Figure 10. Experimental setup.

We used a modified US Air Force resolution test chart for imaging. Number of measurements was set as 600,1200, 1800, 2400, 3000, 3600, and 6400.

The experimental results are shown in Figures 11 and 12, indicating that the imaging quality of the three- and four-step phase-shift FSI improved as the number of measurements increased. The imaging quality of CFSI and the two-step phase-shift FSI increased first and then decreased, which is similar to the simulation using binary patterns. This reason is that FSI using binary patterns after spatial dithering strategy is not able to realize perfect reconstruction [32]. After reaching the best image quality that can be reconstructed by binary patterns, increasing the number of measurements will decrease the imaging quality. CFSI was the first to achieve its best imaging quality, and before that, the PSNR values of CFSI were higher than those of the three other methods.

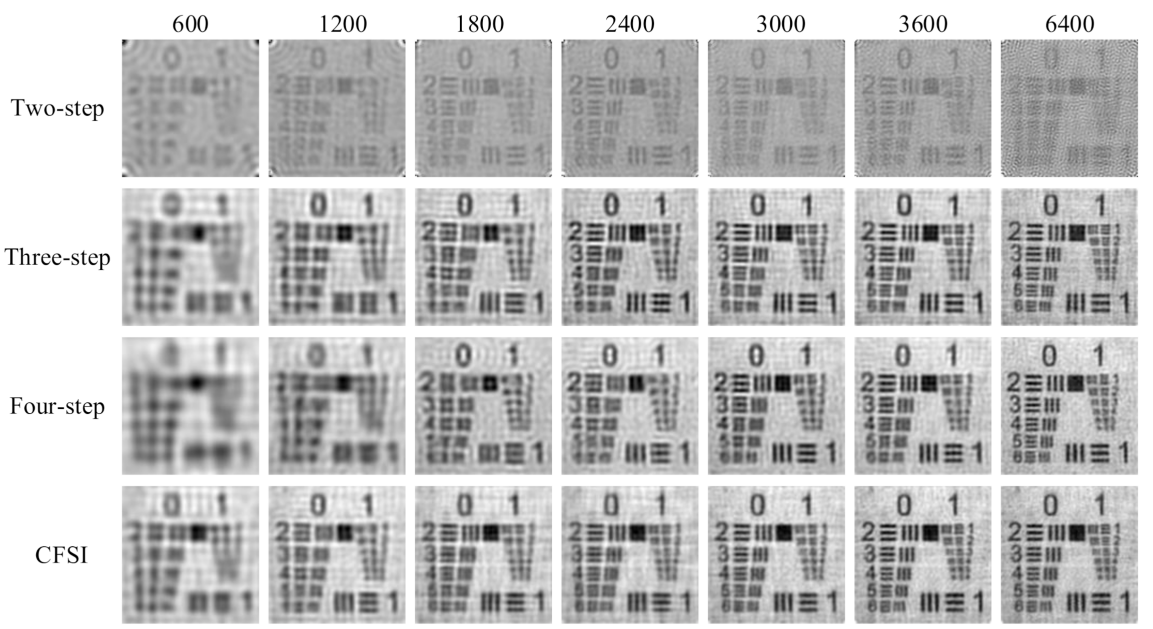

Figure 11. Experimental results of CFSI and two-, three-, and four-step phase-shift FSI under different number of measurements. 


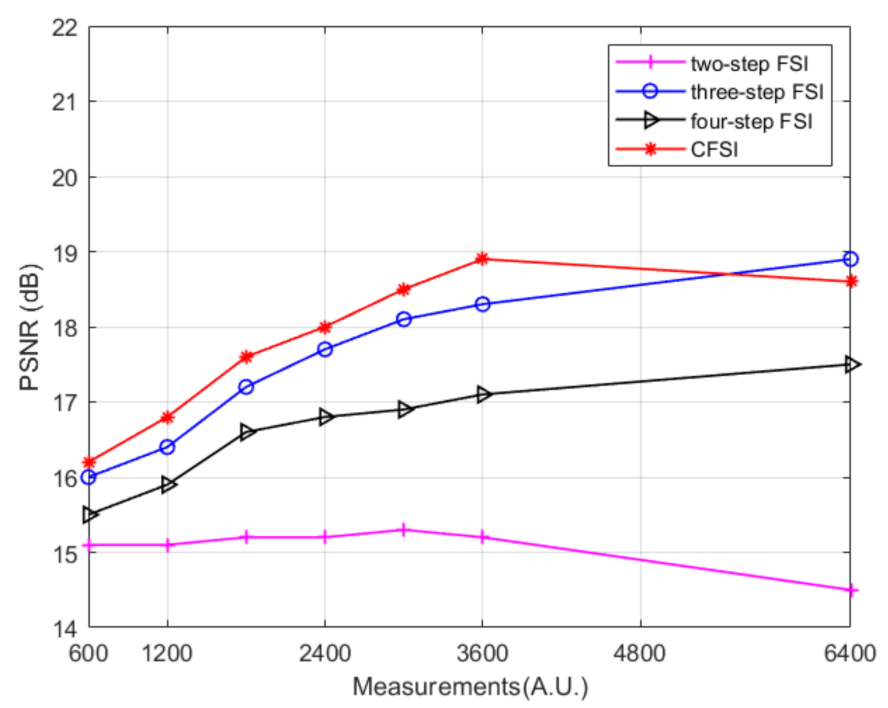

Figure 12. The experimental results are shown in Figures 11 and 12, indicating that the imaging quality of the three- and four-step phase-shift FSI improved as the number of measurements increased.

\section{Discussion and Conclusions}

According to the complementary nature of Fourier patterns based on a four-step phaseshift algorithm and the complementary nature of DMD, CFSI can improve the performance of FSI in terms of noise robustness and imaging efficiency. Conventional FSI does not balance imaging efficiency and imaging quality well. CFSI is a differential measurement based on a four-step phase-shift FSI and has the same imaging efficiency as the two-step phase-shift FSI. The results of simulations and experiments suggest that CFSI has the best imaging quality among the three other methods in an environment with noise under the condition that the best imaging quality of CFSI is not reached. However, CFSI also has several disadvantages. First, complementary patterns after Floyd-Steinberg dithering do not perfectly replace binary patterns with a phase-shift difference of $\pi$ [32]. Using Floyd-Steinberg dithering has a certain effect on the imaging quality of CFSI when the four-step phase-shift formula is used for reconstruction. Second, the complementary region of DMD will introduce unused micromirrors that may cause extra intensity value. In our work, we propose that the unused region should be filled with symmetrical random patterns. Third, in our experiment, we use two detectors to obtain the light intensity value of the complementary patterns. The increase of system complexity is inevitable for collecting the reflected light from different directions of the DMD. But the experimental setup can still be improved to use one detector to collect both the light of complementary patterns, and just need generate a large enough optical path difference by using fibre-optical to distinguish the two intensity value signals of complementary patterns. This method with one detector requires sacrificing the complexity of the imaging system.

The performance of SPI is mainly affected by two aspects, one is sampling and the other is reconstruction. In terms of sampling, although there are many compressed sensing technologies applied to improve the imaging efficiency of SPI [15,16,34-37], the time-consuming reconstruction process limits their application in real-time imaging [27]. Conventional FSI has the advantage of simple reconstruction for only one IFT is required, and it has been applied in real-time and high-quality imaging [29,32]. The reported method CFSI combines the advantages of current methods and improves the performance of conventional FSI. Therefore, CFSI provides an alternative approach to realizing real-time and high-quality imaging. 
Author Contributions: Conceptualization, D.Z. and J.C.; Data curation, D.Z. and H.C.; Formal analysis, D.Z.; Investigation, D.Z., B.-k.C. and K.L.; Methodology, D.Z. and J.C.; Project administration, J.C. and Q.H.; Software, H.C.; Supervision, J.C. and Q.H.; Writing-Original draft, D.Z.; Writing-review \& editing, D.Z. All authors have read and agreed to the published version of the manuscript.

Funding: This research was funded by National Natural Science Foundation of China 61871031, 61875012, and 61905014; Funding of foundation enhancement program under Grant 2020-JCJQ-JJ-030.

Institutional Review Board Statement: Not applicable.

Informed Consent Statement: Not applicable.

Data Availability Statement: The data presented in this study are available upon request from the corresponding author.

Acknowledgments: The authors thank the editor and the anonymous reviewers for their valuable suggestions.

Conflicts of Interest: The authors declare no conflict of interest. The funders had no role in the design of the study; in the collection, analyses, or interpretation of data; in the writing of the manuscript, or in the decision to publish the results.

\section{References}

1. Pittman, T.B.; Shih, Y.H.; Strekalov, D.V.; Sergienko, A.V. Optical imaging by means of two-photon quantum entanglement. Phys. Rev. A 1995, 52, R3429-R3432. [CrossRef] [PubMed]

2. Shapiro, J.H. Computational ghost imaging. Phys. Rev. A 2008, 78, 061802. [CrossRef]

3. Olivieri, L.; Totero Gongora, J.S.; Pasquazi, A.; Peccianti, M. Time-Resolved Nonlinear Ghost Imaging. ACS Photonics 2018, 5, 3379-3388. [CrossRef]

4. Gibson, G.M.; Sun, B.Q.; Edgar, M.P.; Phillips, D.B.; Hempler, N.; Maker, G.T.; Malcolm, G.P.A.; Padgett, M.J. Real-time imaging of methane gas leaks using a single-pixel camera. Opt. Express 2017, 25, 2998-3005. [CrossRef]

5. Khamoushi, S.M.M.; Nosrati, Y.; Tavassoli, S.H. Sinusoidal ghost imaging. Opt. Lett. 2015, 40, 3452-3455. [CrossRef]

6. Radwell, N.; Mitchell, K.J.; Gibson, G.M.; Edgar, M.P.; Bowman, R.; Padgett, M.J. Single-pixel infrared and visible microscope. Optica 2014, 1, 285-289. [CrossRef]

7. Ferri, F.; Magatti, D.; Lugiato, L.A.; Gatti, A. Differential Ghost Imaging. Phys. Rev. Lett. 2010, 104, 253603. [CrossRef]

8. Sun, B.Q.; Welsh, S.S.; Edgar, M.P.; Shapiro, J.H.; Padgett, M.J. Normalized ghost imaging. Opt. Express 2012, 20 , $16892-16901$. [CrossRef]

9. Jiang, H.; Zhu, S.; Zhao, H.; Xu, B.; Li, X. Adaptive regional single-pixel imaging based on the Fourier slice theorem. Opt. Express 2017, 25, 15118-15130. [CrossRef]

10. Devaux, F.; Moreau, P.A.; Denis, S.; Lantz, E. Computational temporal ghost imaging. Optica 2016, 3, 698-701. [CrossRef]

11. Sun, B.; Edgar, M.P.; Bowman, R.; Vittert, L.E.; Welsh, S.; Bowman, A.; Padgett, M.J. 3-D Computational imaging with single-pixel detectors. Science 2013, 340, 844-847. [CrossRef] [PubMed]

12. Sun, M.J.; Edgar, M.P.; Gibson, G.M.; Sun, B.; Radwell, N.; Lamb, R.; Padgett, M.J. Single-pixel three-dimensional imaging with time-based depth resolution. Nat. Commun. 2016, 7, 12010. [CrossRef]

13. Zhang, Z.; Zhong, J. Three-dimensional single-pixel imaging with far fewer measurements than effective image pixels. Opt. Lett. 2016, 41, 2497-2500. [CrossRef]

14. Zhang, Z.; Liu, S.; Peng, J.; Yao, M.; Zheng, G.; Zhong, J. Simultaneous spatial, spectruml, and 3D compressive imaging via efficient Fourier single-pixel measurements. Optica 2018, 5, 315-319. [CrossRef]

15. Watts, C.M.; Shrekenhamer, D.; Montoya, J.; Lipworth, G.; Hunt, J.; Sleasman, T.; Krishna, S.; Smith, D.R.; Padilla, W.J. Terahertz compressive imaging with metamaterial spatial light modulators. Nat. Photonics 2014, 8, 605-609. [CrossRef]

16. Chan, W.L.; Charan, K.; Takhar, D.; Kelly, K.F.; Baraniuk, R.G.; Mittleman, D.M. A single-pixel terahertz imaging system based on compressed sensing. Appl. Phys. Lett. 2008, 93, 121105. [CrossRef]

17. Stantchev, R.I.; Sun, B.Q.; Hornett, S.M.; Hobson, P.A.; Gibson, G.M.; Padgett, M.J.; Hendry, E. Noninvasive, near-field terahertz imaging of hidden objects using a single-pixel detector. Sci. Adv. 2016, 2, e1600190. [CrossRef] [PubMed]

18. Edgar, M.P.; Gibson, G.M.; Bowman, R.W.; Sun, B.; Radwell, N.K.; Mitchell, J.; Welsh, S.S.; Padgett, M.J. Simultaneous real-time visible and infrared video with single-pixel detectors. Sci. Rep. 2015, 5, 10669. [CrossRef] [PubMed]

19. Bian, L.; Suo, J.; Situ, G.; Li, Z.; Fan, J.; Chen, F.; Dai, Q. Multispectral imaging using a single bucket detector. Sci. Rep. 2016, 6, 24752. [CrossRef]

20. Welsh, S.S.; Edgar, M.P.; Bowman, R.; Jonathan, P.; Sun, B.; Padgett, M.J. Fast full-color computational imaging with single-pixel detectors. Opt. Express 2013, 21, 23068-23074. [CrossRef]

21. Satat, G.; Tancik, M.; Gupta, O.; Heshmat, B.; Raskar, R. Object classification through scattering media with deep learning on time resolved measurement. Opt. Express 2017, 25, 17466-27479. [CrossRef] [PubMed] 
22. Katz, O.; Heidmann, P.; Fink, M.; Gigan, S. Non-invasive single-shot imaging through scattering layers and around corners via speckle correlations. Nat. Photonics 2014, 8, 784-790. [CrossRef]

23. Gong, W.; Han, S. Correlated imaging in scattering media. Opt. Lett. 2011, 36, 394-62011. [CrossRef] [PubMed]

24. Huang, J.; Shi, D.; Yuan, K.; Hu, S.; Wang, Y. Computational-weighted Fourier single-pixel imaging via binary illumination. Opt. Express 2018, 26, 16547-16559. [CrossRef] [PubMed]

25. Zhang, Z.; Ma, X.; Zhong, J. Single-pixel imaging by means of Fourier spectrum acquisition. Nat. Commun. 2015, 6, 6225. [CrossRef]

26. Duarte, M.F.; Davenport, M.A.; Takhar, D.; Laska, J.N.; Sun, T.; Kelly, K.F.; Baraniuk, R.G. Single-pixel imaging via compressive sampling. IEEE Signal Process. Mag. 2008, 25, 83-91. [CrossRef]

27. Zhang, Z.; Wang, X.; Zheng, G.; Zhong, J. Hadamard single-pixel imaging versus Fourier single-pixel imaging. Opt. Express 2017, 25, 19619-19639. [CrossRef]

28. Vasile, T.; Damian, V.; Coltuc, D.; Petrovici, M. Single pixel sensing for THz laser beam profiler based on Hadamard Transform. Opt. Laser Technol. 2016, 79, 173-178. [CrossRef]

29. Deng, H.; Gao, X.; Ma, M.; Yao, P.; Guan, Q.; Zhong, X.; Zhang, J. Fourier single-pixel imaging using fewer illumination patterns. Appl. Phys. Lett. 2019, 114, 221906. [CrossRef]

30. Luo, B.; Tsai, K.; Liu, J. Computational ghost imaging by using complementary illumination patterns. In Proceedings of the SPIE 10711: Biomedical Imaging and Sensing Conference, Yokohama, Japan, 25-27 April 2018; p. 107111W.

31. Ye, Z.; Xiong, J.; Liu, H. Ghost Difference Imaging Using One Single-Pixel Detector. Phys. Rev. Appl. 2021, 15, 034035. [CrossRef]

32. Zhang, Z.; Wang, X.; Zheng, G.; Zhong, J. Fast Fourier single-pixel imaging via binary illumination. Sci. Rep. 2017, 7, 12029. [CrossRef] [PubMed]

33. Liu, H.; Yang, B.; Guo, Q.; Shi, J.; Guan, C.; Zheng, G.; Mühlenbernd, H.; Li, G.; Zentgraf, T.; Zhang, S. Single-pixel computational ghost imaging with helicity-dependent metasurface hologram. Sci. Adv. 2017, 3, e1701477. [CrossRef] [PubMed]

34. Takhar, D.; Laska, J.N.; Wakin, M.B.; Duarte, M.F.; Baron, D.; Sarvotham, S.; Kelly, K.F.; Baraniuk, R.G. A new compressive imaging camera architecture using optical-domain compression. In Proceedings of the SPIE 6065: Computational Imaging IV, San Jose, CA, USA, 15-19 January 2006; p. 606509.

35. Horisaki, R.; Matsui, H.; Egami, R.; Tanida, J. Single-pixel compressive diffractive imaging. Appl. Opt. 2017, 56, 1353-1357. [CrossRef]

36. Horisaki, R.; Matsui, H.; Tanida, J. Single-pixel compressive diffractive imaging with structured illumination. Appl. Opt. 2017, 56, 4085-4089. [CrossRef]

37. Clemente, P.; Durán, V.; Tajahuerce, E.; Andrés, P.; Climent, V.; Lancis, J. Compressive holography with a single-pixel detector. Opt. Lett. 2013, 38, 2524-2527. [CrossRef] [PubMed] 